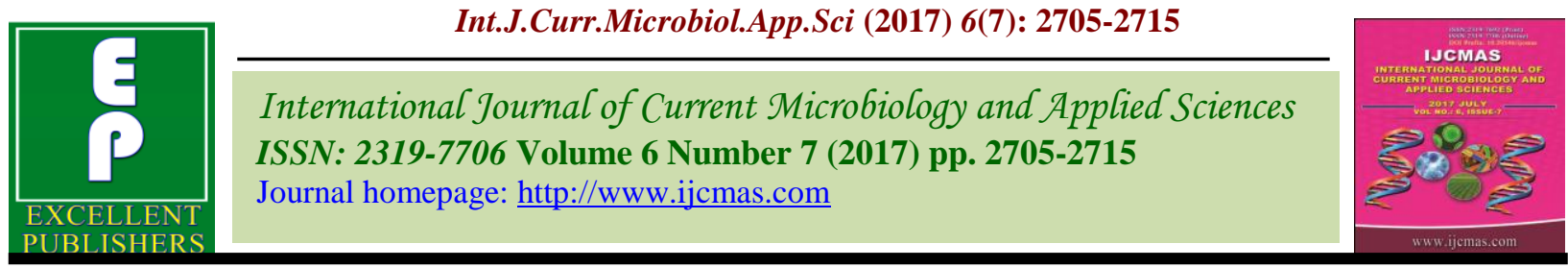

Original Research Article

https://doi.org/10.20546/ijcmas.2017.607.380

\title{
Heterosis Study for Yield, Water Use Efficiency and its Attributing Traits in Greengram (Vigna radiata L. Wilczek)
}

\author{
Sheela Duddagi* and D.L. Savithramma \\ Department of Genetics and Plant Breeding, University of Agricultural Sciences (GKVK), \\ Bengaluru - 560 065, Karnataka, India \\ *Corresponding author
}

\section{A B S T R A C T}

Seven lines, four testers and twenty eight $\mathrm{F}_{1}$ crosses derived through line $\mathrm{x}$ tester

Keywords

Heterosis, WUE, Abiotic stress, L x T, SLA, SCMR

Article Info

Accepted: 29 June 2017 Available Online: 10 July 2017 mating were evaluated to study the magnitude of heterosis for yield, water use efficiency and its attributing traits in order to assess the worth of a cross. The study on extent of heterosis revealed that sixteen hybrids out of twenty eight hybrids recorded high status with maximum score. The hybrid BL $862 \times$ KKM 3 exhibited highest significant negative mid parent heterosis in desirable direction for days to $50 \%$ flowering followed by BL $862 \times$ VGG 4, Hg 19/A $\times$ NP 36 and LM $567 \times$ KKM 3. The hybrid BL $866 \times$ KKM 3 exhibited significant MP and BP heterosis for primary branches per plant, where as BL $862 \times \mathrm{NP} 36$ showed significant mid parent heterosis for clusters per plant. BL $862 \times$ GM $8413(-7.31$ $\%)$, BL $866 \times$ VGG $4(-6.21 \%)$ and BL $862 \times$ GM $8413(-7.35 \%)$, BL $866 \times \mathrm{NP}$ $36(-6.69 \%)$ showed significant negative heterosis over MP and BP for $\Delta{ }^{13} \mathrm{C}$ value, cross SML $134 \times$ VGG $4(51.75 \%)$ revealed positive significant heterosis over better parent for SCMR, these may be useful in future breeding programs.

\section{Introduction}

Mungbean (Vigna radiata (L) Wilczek) $(2 n=2 x=22)$. One of the important pulse crops in India as well as in South East Asia. It is primarily a crop of rainy season. However, with the development of early maturing varieties, it has proved to be an ideal crop for spring and summer seasons. Water stress reduces plant growth and yield. However, water stress that exists at the reproductive stage severely affects grain yield of mungbean more than its occurrence at other stages (Thomas et al., 2004). In addition, the time of flowering and maturity was shortened under stress compared to well water conditions. Progress in crop improvement for water use efficiency was rather slow because a concomitant reduction in total biomass while selecting for increase water use efficiency is perhaps the major reason for the lack of success in plant breeding programme (Kumar et al., 1998). Study of heterosis is important for the plant breeder to find out the superior crosses in first generation itself. In addition to this, the magnitude of heterosis provides basis for determining genetic diversity and also serves as guide to the choice of desirable parents. An attempt was, therefore made to know the magnitude of 
heterosis over mid parent and better parent for seed yield, water use efficiency and its attributing traits in elite Indian mungbean genotypes (Gwande et al., 2001 and Joseph and Santhoshkumar, 2000).

\section{Materials and Methods}

Seven mungbean cultivars based on low $\Delta{ }^{13} \mathrm{C}$ values were selected as lines (BL-866, BL862, Hg19/A, LM-567, ML-347, RMG-62 AND SML-143) were crossed with four high yielding genotypes (NP-36, VGG4, GM-8413 and KKM-3) which were selected as testers based on high $\Delta^{13} \mathrm{C}$ values and were of high yielding in Line $\times$ Tester fashion during kharif 2009-2011 at University of Agricultural Sciences, Bangalore, to generate a total of twenty eight hybrids. All twenty eight hybrids were sown along with their parents in randomized complete block design with two replications and evaluated during rabi 2010. Each entry was sown in $4 \mathrm{~m}$ length with 30 x $10 \mathrm{~cm}$ spacing in single row. Recommended cultural practices were followed.

\section{Specific leaf area (SLA, cm2/g)}

Third fully expanded leaf of the main branch was collected and the leaf area was measured using leaf area meter. Then the leaves were kept in an oven at $70^{\circ} \mathrm{C}$ for 3 days. The dry weight of the leaf was accurately measured using a sensitive balance. SLA was computed using the formula:

$$
\text { SLA }=\frac{\text { Leaf area }(\mathrm{cm} 2)}{\text { Leaf weight }(\mathrm{g})}
$$

\section{SPAD chlorophyll meter reading}

Amongst several leaf characters, leaf thickness and chlorophyll content determines the leaf transmittance characters. Leaf nitrogen content normally influences the leaf chlorophyll content. A device has been developed by Minolta company, New Jersey USA (SPAD-502) which measures the light attenuation at $430 \mathrm{~nm}$ (the peak wavelength for chlorophyll a and chlorophyll b) and at $750 \mathrm{~nm}$ (near infra-red) with no transmittance. The unit value measured by the chlorophyll meter is termed as SCMR (SPAD chlorophyll meter reading) which provides information on the relative amount of leaf chlorophyll. The SPAD meter (Soil Plant Analytical Development) is a simple hand held instrument, which operates with DC power of three volts. The third leaf from the apex was selected to record the SCMR. Selected leaf of green gram was clamped avoiding the mid rib region into the sensor head of SPAD meter. A gentle stroke was given to record the SPAD reading and the average of such four strokes was considered. Since mungbean has trifoliate leaf, SCMR was recorded in all leaflets and the average value wad recorded. The SCMR was recorded under normal sunlight between 9.00 am to $4.00 \mathrm{pm}$.

\section{Mass spectrometric analysis}

Carbon isotope ratios $\left({ }^{13} \mathrm{C}^{12} \quad\right.$ C) in comparison with the Pee and Dee Belemnite standard were measured using continuous flow isotopic mass spectrometer (IRMS). The IRMS facility consists of flash elemental analyzer (CE-EA 1112), for sequential combustion of biomass samples and open slit interference (coulo 3). Finely powdered dry leaves samples were accurately weighed in the range of 1.0 to $1.2 \mathrm{mg}$ into silver capsules. The crimped capsules with the sample were placed sequentially in the caraousel of the auto sampler. The samples are dropped at specific interval of time along with a pulse of pure $\mathrm{O} 2$ in to the oxidation reactor.

The combustion (oxidation) reactor contains chromic oxide and silvered cobaltous- 
cobaltic-oxide heated to $105^{\circ} \mathrm{C}$. The biomass is completely oxidized to produce $\mathrm{CO}_{2}, \mathrm{~N}_{2} \mathrm{O}$ and $\mathrm{H}_{2} \mathrm{O}$. These gases were swept into the reduction furnace using helium carrier gas. The reduction column contains reduced copper in quartz tubes heated to $680^{\circ} \mathrm{C}$. In this reaction, the $\mathrm{N}_{2} \mathrm{O}$ is reduced to $\mathrm{N}_{2}$ and the excess $\mathrm{O}_{2}$ is absorbed. The resultant gases are then flushed through scrubbers to trap $\mathrm{CO}_{2}$ and water.

The pure $\mathrm{CO}_{2}$ and $\mathrm{N}_{2}$ gas after passing through a GC column ( $5^{\circ}$ A molecular sieve) and a thermal conductivity detector (TCD) into the ion source of IRMS. At the source, $\mathrm{CO}_{2}$ is ionized by electron impacts ionization to produce molecular radicals $(\mathrm{CO}+)$. When accelerated radicals pass through a strong magnetic field it is deflected with the radius of deflection being proportional to the molecular mass of the radicals. These deflecting ${ }^{12} \mathrm{CO}_{2}$ and ${ }^{13} \mathrm{CO}_{2}$ are collected by the Faraday cups and the signal is amplified and transmitted to the computer and displayed. Based on fractionation (isotopic composition with respect to $\mathrm{PDB})$, the ${ }^{13} \mathrm{C}$ discrimination $\left(\Delta^{13} \mathrm{C}\right)$ in the plant sample was computed as follows and expressed as parts per thousand (\%) or per mil.

$\Delta{ }^{13} \mathrm{C}(\%)=\frac{\delta_{\mathrm{a}}{ }^{13} \mathrm{C}-\delta_{\mathrm{p}}{ }^{13} \mathrm{C}}{1+\delta_{\mathrm{p}}{ }^{13} \mathrm{C} / 1000}$

All plants in a cross were tagged and observations were recorded on individual plant basis. The observations on water use efficiency, yield and its attributes viz., $\Delta 13 \mathrm{C}$, SLA (Specific leaf area), SCMR (SPAD chlorophyll meter reading), days to $50 \%$ flowering, plant height, primary branches per plant, clusters per plant, pods per cluster, pods per plant, pod yield per plant, pod length, seeds per pod, seed yield per plant, threshing percentage were recorded and subjected to statistical analysis. Improving water use efficiency (WUE) becomes quite relevant among the number of traits suggested for crop improvement under such conditions. The yield model proposed by Passioura (1986):

Seed yield $=$ Water use efficiency (WUE) $\times$ Total transpiration $(\mathrm{T}) \times$ Harvest index $(\mathrm{HI})$

The mean values of the data recorded for water use efficiency, seed yield per plant and their attributing characters in mungbean in each entry of both the replications were first subjected to Analysis of Variance as per the methods outlined by Panse and Sukhatme (1967) using mean values of randomly selected plants. All sources of variation were tested against error for significance by comparing calculated ' $F$ ' value with table ' $F$ ' value at 1 per cent and 5 per cent probability levels.

The treatments mean value for each trait was used for the estimation of heterosis. The per cent heterosis of all $\mathrm{F}_{1}$ crosses over their better parent (BP) and mid parent (MP) were computed by the method suggested by Turner (1953) and Hayes et al., (1955).

\section{Overall gca status of parents, sca status and heterotic status of crosses}

Since yield is associated with several other characters, positively with some and negatively with others, it is necessary to know the overall status of the parents / hybrids considering gca effects / sca effects for all the characters simultaneously. The overall status of a parent or a cross with respect to gca or sca effects was determined as per the method of Arunachalam and Bandyopadhyay (1979) with slight modification as suggested by Mohan Rao (2001). The modified procedure is described as under.

The estimates of general combining ability effects of parents, sca effects and standard 
heterosis of hybrids were ranked by giving the highest rank for the parent or the cross which manifested the highest gca / sca effects and mid parent heterosis respectively in desirable direction. The lowest rank was given for parent or the cross with the lowest gca / sca effects and mid parent heterosis for a character, respectively. This was repeated for each character except days to 50 per cent flowering, for which the ranking was given in reverse order. The ranks obtained by the parent / hybrid were summed up across all the characters to arrive at a total score for each of the parent / cross. Further, the mean of the total scores of all the genotypes (parents and hybrids) was computed which was used as the final norm to ascertain the status of a parent or a hybrid with respect to gca / sca effects and mid parent heterosis. The parent / hybrid whose total rank exceeded the final norm were given high $(\mathrm{H})$ overall gca / sca / heterosis status, respectively. On the other hand, the parents or the cross, whose total rank was less than the final norm were given low (L) overall gca / sca / heterosis status, respectively.

Accordingly the crosses were grouped into different category viz., High $\times$ High $(\mathrm{HH})$, High $\times$ Low $($ HL), Low $\times$ High $(\mathrm{LH})$ and Low $\times$ Low (LL) based on overall gca status of their parents. The overall sca status of crosses viz., High or Low was also mentioned under each category to draw the inference.

\section{Results and Discussion}

Heterosis is usually expressed in the form of increased yield in $\mathrm{F}_{1}$ 's which in turn is dependent on the contribution of many component characters. All the component characters of yield, water use efficiency and its attributing traits were studied for heterosis manifestation in order to assess the worth of a cross. When significant heterosis over better parent is observed in majority of the crosses for any trait, it indicates involvement of nonadditive gene action in the genetic control of that trait. Assuming that epistasis is absent, the cause of heterosis can only be attributed to dominance gene action. The inference drawn about gene action based on heterosis for different characters is presented below based on this assumption.

The extent of heterosis over mid parent (MP) and better parent (BP) expressed by 28 hybrids in respect of grain yield, WUE and its attributing traits have been tabulated in table 1 and briefly presented below trait wise.

The negative heterosis is desirable for days to 50\% flowering. Among 28 crosses, the cross BL $862 \times$ KKM 3 exhibited highest significant negative mid parent heterosis ($31.63 \%$ ) for days to 50 per cent flowering in desirable direction followed by BL $862 \times$ VGG 4 (-28.57\%), Hg 19/A × NP 36 (-23.16 $\%)$ and LM $567 \times$ KKM $3(-22.58 \%)$. Of the above crosses, BL $862 \times$ VGG 4 exhibited maximum significant negative heterosis ($32.04 \%$ ) over better parent followed by LM $567 \times \mathrm{KKM} 3(-22.58 \%)$, and BL $866 \times$ VGG $4(-17.20 \%)$. These findings are in agreement with Naidu and Styanarayan (1993). The cross BL $866 \times$ KKM 3 showed significant positive heterosis over mid parent (114.29 \%) for primary branches per plant. Whereas, BL $866 \times$ KKM 3 recorded highest significant positive heterosis $(114.29 \%)$ over better parent. These findings are in agreement Khattak et al., (2002e).

The cross BL $862 \times$ NP 36 showed significant positive heterosis over mid parent $(87.61 \%)$ for clusters per plant.These findings are in agreement Khattak et al., (2002e).

Four crosses revealed significant heterosis over mid parent for pods per cluster. However only one cross ML $347 \times$ GM 8413 showed significant positive heterosis over mid parent 
(72.22\%). Whereas, four crosses revealed significant heterosis over better parent these findings are in agreement with Khattak et al., (2002e). However none of cross showed positive heterosis over better parent.

12 crosses revealed significant heterosis over mid parent for pods per plant. Only one cross RMG $62 \times$ VGG 4 showed significant positive heterosis over mid parent $(25.23 \%)$ (Khattak et al., 2002e).

Whereas, 11 crosses revealed significant heterosis over better parent among that cross $\mathrm{Hg}$ 19/A $\times$ GM 8413 recorded highest significant negative heterosis $(54.10 \%)$ over better parent.

19 crosses revealed significant heterosis over mid parent for seeds per pod. Only one cross BL $866 \times$ NP 36 showed significant positive heterosis over mid parent (19.15\%).

Whereas, 20 hybrids revealed significant heterosis over better parent among that none of hybrid revealed positive heterosis over better parent (Khattak et al., 2002e and Joseph and Santhoshkumar, 2000).

Four crosses revealed significant heterosis over mid parent for pod length $(\mathrm{cm})$. Only one cross SML $134 \times$ VGG 4 showed significant positive heterosis over mid parent $(27.83 \%)$. Whereas, seven hybrids revealed significant heterosis over better parent among that only one cross revealed positive heterosis over better parent (27.08).

15 crosses revealed significant heterosis over mid parent pod yield per plant (g). None of the crosses showed significant positive heterosis over mid parent. Whereas, 18 crosses revealed significant heterosis over better parent none of the crosses revealed positive heterosis over better parent. Eight crosses revealed significant heterosis over mid parent for seed yield per plant $(\mathrm{g})$. None of the crosses showed significant positive heterosis over mid parent. Whereas, 19 crosses revealed significant heterosis over better parent none of the crosses revealed positive heterosis over better parent (Dethe and Patil, 2008 and Khattak et al., 2002e).

Four crosses revealed significant heterosis over mid parent for threshing percentage. Out of that three crosses showed significant positive heterosis over mid parent viz., RMG $62 \times$ KKM 3 (25.97\%), followed by RMG 62 $\times \mathrm{NP} 36(23.30 \%)$ and LM $567 \times$ VGG 4 $(17.16 \%)$. Whereas, five crosses revealed significant heterosis over better parent out of that two crosses revealed positive heterosis over better parent viz., RMG $62 \times$ KKM 3 (24.05\%), followed by RMG $62 \times \mathrm{NP} 36$ $(21.95 \%)$.

10 crosses revealed significant heterosis over mid parent for test weight $(\mathrm{g})$. Out of that 4 crosses showed significant positive heterosis over mid parent viz., BL $866 \times$ KKM 3 (21.83 $\%$ ), followed by BL $862 \times$ GM 8413 (13.30 $\%)$, BL $866 \times$ NP $36(8.50 \%)$ and LM $567 \times$ GM $8413(8.05 \%)$.

Whereas, ten crosses revealed significant heterosis over better parent out of that 2 crosses revealed positive heterosis over better parent viz., BL $866 \times \mathrm{KKM} 3(15.86 \%)$, followed by BL $862 \times$ GM 8413 (12.30\%).

Three crosses revealed significant heterosis over mid parent for $\Delta{ }^{13} \mathrm{C}$ value. Out of that two crosses showed significant negative heterosis over mid parent viz., BL $862 \times \mathrm{GM}$ $8413(-7.31 \%)$, followed by BL $866 \times$ VGG $4(-6.21 \%)$.

Whereas, three crosses revealed significant heterosis over better parent out of that two crosses revealed negative heterosis over better parent viz., BL $862 \times$ GM $8413(-7.35 \%)$ followed by BL $866 \times$ NP $36(-6.69 \%)$. 
Table.1 Estimates of mid parent and better parent (BP) heterosis in hybrids for growth, yield and WUE related traits in mungbean

\begin{tabular}{|c|c|c|c|c|c|c|c|c|c|c|c|c|}
\hline \multirow{3}{*}{ Hybrids } & \multirow{2}{*}{\multicolumn{2}{|c|}{$\begin{array}{c}\begin{array}{c}\text { Days to 50 \% } \\
\text { flowering }\end{array} \\
\text { Heterosis } \\
\end{array}$}} & \multirow{2}{*}{\multicolumn{2}{|c|}{$\begin{array}{c}\begin{array}{c}\text { Plant height } \\
(\mathrm{cm})\end{array} \\
\text { Heterosis }\end{array}$}} & \multirow{2}{*}{\multicolumn{2}{|c|}{$\begin{array}{c}\text { Primary branches per plant } \\
\text { Heterosis }\end{array}$}} & \multirow{2}{*}{\multicolumn{2}{|c|}{$\begin{array}{c}\text { Clusters per plant } \\
\text { Heterosis }\end{array}$}} & \multirow{2}{*}{\multicolumn{2}{|c|}{$\begin{array}{c}\begin{array}{c}\text { Pods per } \\
\text { cluster }\end{array} \\
\text { Heterosis } \\
\end{array}$}} & \multirow{2}{*}{\multicolumn{2}{|c|}{$\begin{array}{c}\begin{array}{c}\text { Pods per } \\
\text { plant }\end{array} \\
\text { Heterosis }\end{array}$}} \\
\hline & & & & & & & & & & & & \\
\hline & MP & BP & MP & BP & MP & BP & MP & BP & MP & BP & MP & BP \\
\hline RMG $62 \times$ GM8413 & 4.49 & 2.20 & 4.54 & 3.80 & 12.50 & 0.00 & 7.56 & -4.48 & 18.75 & 5.56 & 17.39 & -3.57 \\
\hline RMG $62 \times$ KKM 3 & $-5.56 *$ & $-8.60 * *$ & 12.53 & 4.37 & 37.50 & 22.22 & 23.97 & 11.94 & -29.73 & -31.58 & -11.32 & -16.07 \\
\hline RMG $62 \times$ NP 36 & 3.41 & 2.25 & -13.31 & $-20.24 * *$ & -22.22 & -22.22 & -14.53 & -25.37 & 17.95 & 9.52 & 6.67 & 0.00 \\
\hline RMG $62 \times$ VGG 4 & $-7.78 * *$ & $-10.75 * *$ & $34.34 * *$ & 12.68 & 25.00 & 11.11 & 36.09 & 17.91 & -12.20 & -21.74 & $25.23 *$ & 19.64 \\
\hline BL $866 \times$ GM 8413 & $-9.71 * *$ & $-13.19 * *$ & $-18.96 *$ & $-22.43 *$ & 14.29 & 14.29 & -20.14 & -25.86 & 37.93 & 33.33 & 14.29 & 7.32 \\
\hline BL $866 \times$ KKM 3 & $-7.34 * *$ & $-11.83 * *$ & $19.49 *$ & 16.41 & $114.29 * *$ & $114.29 * *$ & 36.18 & 28.67 & -17.65 & -26.32 & -3.30 & -12.00 \\
\hline BL $866 \times$ NP 36 & -1.73 & -4.49 & 10.84 & -2.60 & -12.50 & -22.22 & 35.50 & 23.56 & $-50.00 *$ & $-57.14 *$ & $-40.00 * *$ & $-44.90 * *$ \\
\hline BL $866 \times$ VGG 4 & $-12.99 * *$ & $-17.20 * *$ & -5.26 & -17.03 & 0.00 & 0.00 & 0.18 & -9.39 & -36.84 & $-47.83 *$ & $-30.43 *$ & $-37.25 * *$ \\
\hline ML 347 × GM 8413 & $-7.94 * *$ & $-11.22 * *$ & 1.33 & -7.29 & 6.67 & 0.00 & -30.69 & -32.69 & $72.22 * *$ & 40.91 & 22.73 & 3.85 \\
\hline ML $347 \times$ KKM 3 & $-13.09 * *$ & $-15.31 * *$ & $30.13 * *$ & $27.35 * *$ & 20.00 & 12.50 & 61.75 & 54.26 & -31.71 & -36.36 & 9.80 & 7.69 \\
\hline ML $347 \times$ NP 36 & $-10.16 * *$ & $-14.29 * *$ & 13.74 & -4.02 & -17.65 & -22.22 & -4.04 & -5.00 & -11.63 & -13.64 & -16.83 & -19.23 \\
\hline ML $347 \times$ VGG 4 & $-13.09 * *$ & $-15.31 * *$ & $31.83 * *$ & 20.48 & 46.67 & 37.50 & -8.26 & -8.35 & 15.56 & 13.04 & 2.91 & 1.92 \\
\hline LM $567 \times$ GM 8413 & $-7.61 * *$ & $-8.60 * *$ & $-21.25 *$ & $-28.00 * *$ & -28.57 & -28.57 & 23.33 & 15.38 & -35.48 & -41.18 & $-30.67 *$ & -33.33 \\
\hline LM $567 \times$ KKM 3 & $-22.58 * *$ & $-22.58 * *$ & $-28.74 * *$ & $-30.33 * *$ & -14.29 & -14.29 & 23.06 & 13.15 & -33.33 & -36.84 & $-25.84 *$ & $-34.00 *$ \\
\hline LM $567 \times$ NP 36 & -3.30 & -5.38 & $-39.09 * *$ & $-48.64 * *$ & 0.00 & -11.11 & -9.13 & -13.40 & -15.79 & -23.81 & -11.36 & -20.41 \\
\hline LM $567 \times$ VGG 4 & 0.00 & 0.00 & $53.63 * *$ & $40.52 * *$ & 42.86 & 42.86 & $85.38 *$ & 78.21 & $-60.00 * *$ & $-65.22 * *$ & $-35.56 * *$ & $-43.14 * *$ \\
\hline SML $134 \times$ GM 8413 & $-5.21 *$ & $-9.90 * *$ & $-23.77 * *$ & $-27.86 * *$ & 20.00 & 12.50 & 8.16 & 5.77 & -14.29 & -28.57 & -10.11 & -24.53 \\
\hline
\end{tabular}

Table.1 (Continued)

\begin{tabular}{|c|c|c|c|c|c|c|c|c|c|c|c|c|}
\hline 1 & 2 & 3 & 4 & 5 & 6 & 7 & 8 & 9 & 10 & 11 & 12 & 13 \\
\hline SML $134 \times$ KKM 3 & $-5.15 *$ & $-8.91 * *$ & -13.96 & -15.20 & 6.67 & 0.00 & -13.21 & -16.67 & -20.00 & -23.81 & $-30.10 * *$ & $-32.08 *$ \\
\hline SML $134 \times$ NP 36 & -1.05 & $-6.93 *$ & $-15.65 *$ & $-26.63 * *$ & 5.88 & 0.00 & -19.76 & -20.00 & 19.05 & 19.05 & -1.96 & -5.66 \\
\hline SML $134 \times$ VGG 4 & $-5.15 *$ & $-8.91 * *$ & $19.35 *$ & 5.60 & -6.67 & -12.50 & -6.88 & -7.44 & 22.73 & 17.39 & 15.38 & 13.21 \\
\hline BL $862 \times$ GM8413 & $-12.37 * *$ & $-17.48 * *$ & $-35.34 * *$ & $-38.57 * *$ & 7.69 & 0.00 & 38.48 & 38.08 & -2.86 & -19.05 & -8.70 & $-25.00 *$ \\
\hline BL $862 \times$ KKM 3 & $-31.63 * *$ & $-34.95 * *$ & $-31.90 * *$ & $-33.14 * *$ & 38.46 & 28.57 & -1.42 & -3.52 & -35.00 & -38.10 & $-33.96 * *$ & $-37.50 * *$ \\
\hline BL $862 \times$ NP 36 & $-13.02 * *$ & $-18.93 * *$ & $-33.56 * *$ & $-42.01 * *$ & 6.67 & -11.11 & $87.61 *$ & 84.53 & -28.57 & -28.57 & -4.76 & -10.71 \\
\hline BL $862 \times$ VGG 4 & $-28.57 * *$ & $-32.04 * *$ & $-27.43 * *$ & $-36.02 * *$ & 7.69 & 0.00 & 14.09 & 11.22 & -36.36 & -39.13 & $-30.84 * *$ & $-33.93 * *$ \\
\hline Hg 19/A $\times$ GM 8413 & $-8.38 * *$ & $-9.89 * *$ & $-46.12 * *$ & $-49.49 * *$ & 14.29 & 14.29 & -48.53 & $-58.33 *$ & 14.29 & 14.29 & $-42.27 * *$ & $-54.10 * *$ \\
\hline Hg 19/A $\times$ KKM 3 & $-9.39 * *$ & $-11.83 * *$ & $-21.25 * *$ & $-30.75 * *$ & 14.29 & 14.29 & 19.57 & -1.79 & -21.21 & -31.58 & -9.91 & -18.03 \\
\hline $\mathrm{Hg} \mathrm{19/A} \mathrm{\times} \mathrm{NP} 36$ & $-23.16 * *$ & $-23.60 * *$ & $-28.13 * *$ & $-30.05 * *$ & -25.00 & -33.33 & -15.37 & -32.50 & -14.29 & -28.57 & $-21.82 *$ & $-29.51 * *$ \\
\hline Hg 19/A x VGG 4 & $-8.29 * *$ & $-10.75 * *$ & -16.00 & $-32.75 * *$ & 42.86 & 42.86 & 11.95 & -11.31 & $-45.95 *$ & $-56.52 * *$ & $-37.50 * *$ & $-42.62 * *$ \\
\hline S.Em. \pm & 1.20 & 1.39 & 2.64 & 3.05 & 0.87 & 1.01 & 0.95 & 1.10 & 2.10 & 2.42 & 2.80 & 3.24 \\
\hline
\end{tabular}

*Significant at $\mathrm{P}=0.05$ level **Significant at $\mathrm{P}=0.01$ level 
Table.1 (Continued)

\begin{tabular}{|c|c|c|c|c|c|c|c|c|c|c|c|c|}
\hline \multirow{3}{*}{ Hybrids } & \multirow{2}{*}{\multicolumn{2}{|c|}{$\begin{array}{c}\text { Seeds per pod } \\
\text { Heterosis }\end{array}$}} & \multirow{2}{*}{\multicolumn{2}{|c|}{$\begin{array}{c}\text { Pod length }(\mathrm{cm}) \\
\text { Heterosis }\end{array}$}} & \multirow{2}{*}{\multicolumn{2}{|c|}{$\begin{array}{c}\begin{array}{c}\text { Pod yield per } \\
\text { plant }(\mathrm{g})\end{array} \\
\text { Heterosis }\end{array}$}} & \multirow{2}{*}{\multicolumn{2}{|c|}{$\begin{array}{c}\begin{array}{c}\text { Seed yield per plant } \\
(\mathrm{g})\end{array} \\
\text { Heterosis }\end{array}$}} & \multirow{2}{*}{\multicolumn{2}{|c|}{$\begin{array}{c}\text { Threshing \% } \\
\text { Heterosis }\end{array}$}} & \multirow{2}{*}{\multicolumn{2}{|c|}{$\begin{array}{c}\text { Test weight (g) } \\
\text { Heterosis }\end{array}$}} \\
\hline & & & & & & & & & & & & \\
\hline & MP & BP & MP & $\mathbf{B P}$ & MP & $\mathbf{B P}$ & MP & $\mathbf{B P}$ & MP & BP & MP & BP \\
\hline RMG $62 \times$ GM8413 & -9.52 & -9.52 & -1.27 & -13.38 & $-19.55 *$ & $-31.39 * *$ & -12.74 & $-25.39 *$ & 8.61 & 8.31 & -4.70 & $-8.47 *$ \\
\hline RMG $62 \times$ KKM 3 & $-20.18 *$ & $-23.65 *$ & -4.03 & -12.15 & $-31.01 * *$ & $-37.52 * *$ & -13.51 & $-22.72 *$ & $25.97 * *$ & $24.05 *$ & $-8.51 *$ & $-8.83 *$ \\
\hline RMG $62 \times$ NP 36 & -13.95 & -15.91 & -12.91 & $-21.15 *$ & $-28.00 * *$ & $-32.37 * *$ & -11.54 & -15.98 & $23.30 * *$ & $21.95 *$ & $-8.08 *$ & $-13.89 * *$ \\
\hline RMG $62 \times$ VGG 4 & -10.60 & -12.64 & -5.55 & -15.62 & $-22.32 *$ & $-29.17 * *$ & -16.78 & $-23.10 *$ & 6.62 & 5.14 & $-17.65 * *$ & $-18.33 * *$ \\
\hline BL $866 \times$ GM 8413 & $-32.22 * *$ & $-37.64 * *$ & 13.99 & 0.00 & -18.02 & $-28.54 * *$ & $-26.74 *$ & $-34.44 * *$ & -11.27 & -13.89 & -5.24 & -5.88 \\
\hline BL $866 \times$ KKM 3 & -12.29 & -15.80 & 15.88 & 6.08 & -0.80 & -8.02 & 6.35 & -0.28 & 7.96 & 6.67 & $21.83 * *$ & $15.86 * *$ \\
\hline BL $866 \times$ NP 36 & $19.15 *$ & $-24.00 * *$ & -15.46 & $-23.46 *$ & $-37.49 * *$ & $-39.82 * *$ & $-33.70 * *$ & $-33.72 * *$ & 5.56 & 1.63 & $8.50 *$ & 6.42 \\
\hline BL $866 \times$ VGG 4 & $-32.98 * *$ & $-37.00 * *$ & -14.77 & $-23.85 *$ & $-36.85 * *$ & $-41.04 * *$ & $-46.73 * *$ & $-48.24 * *$ & $-16.25 *$ & $-19.60 *$ & 1.91 & -1.98 \\
\hline ML $347 \times$ GM 8413 & $-22.18 *$ & $-25.57 * *$ & 1.02 & -7.39 & 8.09 & -6.91 & -0.95 & -14.59 & -8.47 & -8.61 & 3.14 & -3.47 \\
\hline ML $347 \times$ KKM 3 & $-32.04 * *$ & $-32.04 * *$ & -10.77 & -14.44 & -8.69 & -16.42 & -16.26 & $-24.51 *$ & -7.42 & -8.95 & 2.84 & -7.59 \\
\hline ML $347 \times$ NP 36 & $-24.44 * *$ & $-26.09 * *$ & -7.22 & -12.06 & -16.66 & $-20.84 *$ & -15.74 & -19.20 & 1.52 & 0.55 & -0.75 & -4.77 \\
\hline ML $347 \times$ VGG 4 & $-47.78 * *$ & $-48.91 * *$ & -16.18 & -21.67 & -2.34 & -9.99 & -5.32 & -11.69 & -3.46 & -4.68 & $-9.80 *$ & $-18.08 * *$ \\
\hline LM $567 \times$ GM 8413 & $-19.30 *$ & $-21.14 *$ & 14.19 & 9.07 & $-23.93 *$ & $-25.95 *$ & -20.28 & -20.42 & 4.61 & 2.03 & $8.05 *$ & 4.05 \\
\hline LM $567 \times$ KKM 3 & $-36.80 * *$ & $-38.17 * *$ & $-26.10 *$ & $-32.50 *$ & $-22.42 *$ & $-25.58 *$ & -22.13 & $-25.81 *$ & 0.09 & -0.62 & $-11.17 * *$ & $-11.72 * *$ \\
\hline LM $567 \times$ NP 36 & $-30.45 * *$ & $-30.45 * *$ & 8.58 & 0.28 & -19.88 & $-25.97 *$ & -17.36 & $-25.95 *$ & 3.40 & 0.04 & -4.24 & $-10.06 *$ \\
\hline LM $567 \times$ VGG 4 & $-31.86 * *$ & $-31.86 * *$ & 8.14 & 1.27 & $-31.31 * *$ & $-34.58 * *$ & -19.40 & $-25.84 *$ & $17.16 *$ & 13.03 & 0.98 & 0.42 \\
\hline SML $134 \times$ GM 8413 & -5.85 & -10.33 & 5.82 & 4.25 & $-28.51 * *$ & $-34.42 * *$ & -20.60 & $-27.47 *$ & 10.41 & 9.82 & 1.49 & -4.03 \\
\hline SML $134 \times$ KKM 3 & -14.29 & $-21.74 *$ & -9.90 & -12.78 & $-44.94 * *$ & $-46.05 * *$ & $-40.56 * *$ & $-43.04 * *$ & 7.85 & 5.37 & -4.70 & -5.91 \\
\hline SML $134 \times$ NP 36 & $-24.39 *$ & $-29.55 * *$ & -0.73 & -2.75 & -7.91 & -9.66 & -12.46 & -14.45 & -4.41 & -4.69 & 3.13 & -4.84 \\
\hline SML $134 \times$ VGG 4 & $-22.29 *$ & $-27.59 * *$ & $27.83 *$ & $27.08 *$ & 9.61 & 8.20 & 7.58 & 6.92 & -1.82 & -2.40 & 1.38 & -1.08 \\
\hline BL $862 \times$ GM8413 & -16.67 & -16.67 & 11.65 & 6.58 & -9.02 & -18.02 & -19.07 & $-30.73 * *$ & -11.08 & $-16.59 *$ & $13.30 * *$ & $12.30 * *$ \\
\hline BL $862 \times$ KKM 3 & $-33.82 * *$ & $-36.70 * *$ & -7.36 & -7.41 & $-21.48 *$ & $-24.54 *$ & $-33.38 * *$ & $-40.41 * *$ & -14.55 & $-21.18 * *$ & 1.14 & -2.34 \\
\hline BL $862 \times$ NP 36 & 0.00 & -2.27 & 0.33 & -0.83 & -12.98 & -13.03 & \begin{tabular}{|l|}
-16.45 \\
\end{tabular} & $-20.55 *$ & -5.32 & -10.50 & 3.99 & \begin{tabular}{|l|}
0.44 \\
\end{tabular} \\
\hline BL $862 \times$ VGG 4 & $-32.88 * *$ & $-34.41 * *$ & -8.18 & -10.57 & $-39.60 * *$ & $-41.53 * *$ & $-38.81 * *$ & $-43.39 * *$ & 1.61 & -3.68 & $-11.93 * *$ & $-13.98 * *$ \\
\hline Hg 19/A × GM 8413 & $-38.10 * *$ & $-38.10 * *$ & $-33.24 * *$ & $-38.78 * *$ & -12.26 & -20.71 & -6.88 & -17.41 & 6.51 & 3.78 & -6.08 & -6.57 \\
\hline Hg 19/A $\times$ KKM 3 & $-35.27 * *$ & $-38.09 * *$ & -13.30 & -16.84 & -14.83 & -17.89 & -17.04 & $-22.94 *$ & -2.94 & -7.07 & -1.36 & -5.10 \\
\hline Hg 19/A × NP 36 & $-33.91 * *$ & $-35.41 * *$ & $-36.32 * *$ & $-39.63 * *$ & $-22.46 *$ & $-22.67 *$ & $-32.81 * *$ & $-33.47 * *$ & -13.11 & -14.65 & -4.70 & -7.61 \\
\hline Hg 19/A × VGG 4 & 6.98 & 4.55 & 0.05 & -6.46 & $-25.50 *$ & $-27.65 *$ & $-31.98 * *$ & $-34.55 * *$ & -9.46 & -10.80 & 4.06 & 1.27 \\
\hline S.Em. \pm & 0.93 & 1.08 & 0.57 & 0.66 & 1.11 & 1.29 & 0.70 & 0.81 & 4.57 & 5.28 & 0.12 & 0.14 \\
\hline
\end{tabular}

*Significant at $\mathrm{P}=0.05$ level **Significant at $\mathrm{P}=0.01$ level 
Table.1 (Continued)

\begin{tabular}{|c|c|c|c|c|c|c|}
\hline \multirow{3}{*}{ Hybrids } & \multirow{2}{*}{\multicolumn{2}{|c|}{$\begin{array}{c}\Delta^{13} \mathrm{C} \\
\text { Heterosis }\end{array}$}} & \multirow{2}{*}{\multicolumn{2}{|c|}{$\begin{array}{c}\text { SLA }\left(\mathrm{cm}^{2} / \mathrm{g}\right) \\
\text { Heterosis }\end{array}$}} & \multirow{2}{*}{\multicolumn{2}{|c|}{$\begin{array}{c}\text { SCMR } \\
\text { Heterosis }\end{array}$}} \\
\hline & & & & & & \\
\hline & MP & $\mathbf{B P}$ & MP & $\mathbf{B P}$ & MP & $\mathbf{B P}$ \\
\hline RMG $62 \times$ GM8413 & -1.84 & -3.34 & 0.34 & -4.71 & 9.30 & 3.10 \\
\hline RMG $62 \times$ KKM 3 & -0.84 & -2.07 & -12.24 & -21.25 & -6.33 & -13.85 \\
\hline RMG $62 \times$ NP 36 & -2.20 & -3.16 & -1.06 & -6.41 & -10.25 & -15.98 \\
\hline RMG $62 \times$ VGG 4 & -1.71 & -2.68 & -24.43 & -24.55 & 15.81 & 11.90 \\
\hline BL $866 \times$ GM 8413 & -4.93 & -5.35 & 38.10 & 25.44 & $53.63 *$ & 30.33 \\
\hline BL $866 \times$ KKM 3 & -0.23 & -2.53 & -3.47 & -23.57 & $53.67 *$ & -1.61 \\
\hline BL $866 \times$ NP 36 & -4.72 & $-6.69 *$ & 21.87 & 11.12 & 10.29 & 4.82 \\
\hline BL $866 \times$ VGG 4 & $-6.21 *$ & -6.32 & 2.44 & -11.04 & -13.89 & -20.66 \\
\hline ML $347 \times$ GM 8413 & -2.19 & -3.96 & -12.88 & -16.93 & 52.27 & 51.65 \\
\hline ML $347 \times$ KKM 3 & 0.46 & -0.50 & 4.53 & -6.55 & 5.42 & 2.22 \\
\hline ML $347 \times$ NP 36 & 0.81 & 0.10 & 0.55 & -4.51 & 10.75 & -1.45 \\
\hline ML 347 × VGG 4 & -2.84 & -4.08 & -9.76 & -10.00 & 8.78 & -0.28 \\
\hline LM $567 \times$ GM 8413 & -3.20 & -5.90 & 38.87 & 26.10 & -11.94 & -15.75 \\
\hline LM $567 \times$ KKM 3 & 2.40 & 2.34 & -1.36 & -21.91 & 34.48 & 32.10 \\
\hline LM $567 \times$ NP 36 & 2.72 & 2.40 & 1.79 & -7.21 & 13.56 & -3.11 \\
\hline LM $567 \times$ VGG 4 & 1.69 & -0.61 & 13.34 & -1.59 & 15.73 & 1.56 \\
\hline SML $134 \times$ GM 8413 & -2.47 & -4.01 & -4.30 & -17.27 & 26.84 & 13.36 \\
\hline SML $134 \times$ KKM 3 & -1.06 & -2.24 & -31.47 & -32.13 & 38.83 & 21.18 \\
\hline SML $134 \times$ NP 36 & $12.12 * *$ & $11.06 * *$ & 20.47 & 3.77 & -42.38 & -42.90 \\
\hline SML $134 \times$ VGG 4 & -0.55 & -1.58 & -19.80 & -27.51 & $55.44 *$ & $51.75 *$ \\
\hline BL $862 \times$ GM8413 & $-7.31 *$ & $-7.35 *$ & 2.13 & -7.73 & 6.74 & 5.75 \\
\hline BL $862 \times$ KKM 3 & -0.23 & -2.91 & -24.98 & -29.27 & 27.76 & 23.25 \\
\hline BL $862 \times$ NP 36 & 3.31 & 0.78 & 0.13 & -9.88 & -14.15 & -23.25 \\
\hline BL $862 \times$ VGG 4 & -3.52 & -4.01 & -13.32 & -17.91 & 18.56 & 41.55 \\
\hline Hg 19/A × GM 8413 & -4.31 & -5.14 & 15.20 & 11.33 & 4.07 & 2.39 \\
\hline Hg 19/A × KKM 3 & 0.87 & -2.72 & $61.71 * *$ & 34.76 & -17.58 & -21.03 \\
\hline Hg 19/A x NP 36 & 1.81 & -1.56 & 35.49 & 31.48 & 19.71 & 7.69 \\
\hline Hg 19/A x VGG 4 & -1.83 & -3.21 & -3.08 & -4.71 & -21.52 & -27.24 \\
\hline S.Em. \pm & 0.61 & 0.70 & 30.73 & 35.48 & 8.79 & 10.15 \\
\hline
\end{tabular}

*Significant at $\mathrm{P}=0.05$ level

**Significant at $\mathrm{P}=0.01$ level 
Table.2 Overall heterotic status of hybrids in mungbean

\begin{tabular}{|c|c|c|c|c|c|}
\hline $\begin{array}{c}\text { Testers } \\
\text { Lines }\end{array}$ & $\begin{array}{c}\text { GM 8413 } \\
\text { (H) }\end{array}$ & $\begin{array}{c}\text { KKM } 3 \\
\text { (H) }\end{array}$ & $\begin{array}{c}\text { NP 36 } \\
\text { (H) }\end{array}$ & $\begin{array}{c}\text { VGG } 4 \\
\text { (L) }\end{array}$ & Hybrids with OHHS \\
\hline LM $567(\mathrm{~L})$ & $(240.5) \mathrm{H}$ & $(275) \mathrm{H}$ & $(259.5) \mathrm{H}$ & $(225.5) \mathrm{H}$ & 4 \\
\hline SML 134 (L) & $(183) \mathrm{L}$ & $(240.5) \mathrm{H}$ & (242) H & $(142.5) \mathrm{L}$ & 2 \\
\hline ML 347 (L) & (149) L & (194) L & (221) H & $(182.5) \mathrm{L}$ & 1 \\
\hline RMG $62(\mathrm{H})$ & (190) L & (202) L & $(229) \mathrm{H}$ & $(157) \mathrm{L}$ & 1 \\
\hline BL $866(L)$ & (207) L & $(113.5) \mathrm{L}$ & $(249) \mathrm{H}$ & $(283.5) \mathrm{H}$ & 2 \\
\hline Hg 19/A (L) & $(260.5) \mathrm{H}$ & $(264) \mathrm{H}$ & $(303.5) \mathrm{H}$ & $(232.5) \mathrm{H}$ & 4 \\
\hline BL $862(\mathrm{H})$ & $(193.5) \mathrm{L}$ & $(239.5) \mathrm{H}$ & $(206) \mathrm{L}$ & $(241.5) \mathrm{H}$ & 2 \\
\hline Hybrids with OHHS & 2 & 4 & 6 & 4 & 16 \\
\hline
\end{tabular}

Final Norm 218.82

(H) : Over all high general combiner; (L) :

Over all low general combiner

$\mathrm{H}$ : Over all high specific combiner; L :

Over all low specific combiner

OHHS : Overall high heterotic status

Table.3 Distribution of heterotic crosses in relation to overall gca and sca status of parents and hybrids in mungbean

\begin{tabular}{|l|c|c|c|c|c|}
\hline $\begin{array}{c}\text { Parental } \\
\text { gca }\end{array}$ & $\begin{array}{c}\text { No. of crosses } \\
\text { under } \\
\text { category }\end{array}$ & $\begin{array}{c}\text { No. of crosses with } \\
\text { high overall sca } \\
\text { status }\end{array}$ & $\begin{array}{c}\text { No. of crosses } \\
\text { with high } \\
\text { heterotic status }\end{array}$ & $\begin{array}{c}\text { Conditional probability } \\
\text { of given cross belonging } \\
\text { to high sca status }\end{array}$ & $\begin{array}{c}\text { Conditional probability of } \\
\text { given cross belonging to high } \\
\text { heterotic status }\end{array}$ \\
\hline H x H & 6 & 5 & 2 & 0.31 & 0.13 \\
\hline H x L & 2 & 1 & 1 & 0.06 & 0.06 \\
\hline L x H & 15 & 8 & 10 & 0.50 & 0.63 \\
\hline L x L & 5 & 2 & 3 & 0.13 & 0.19 \\
\hline
\end{tabular}


Only one cross Hg 19/A × KKM 3 (61.71\%) revealed positive significant heterosis over mid parent for SLA $\left(\mathrm{cm}^{2} / \mathrm{g}\right)$. Whereas, none of the crosses revealed significant heterosis over better parent.

Three crosses showed significant positive heterosis over mid parent for SCMR. viz., SML $134 \times$ VGG 4 (55.54\%) followed by BL $866 \times$ KKM $3(53.67 \%)$ and LM $567 \times$ GM 8413(8.05 \%) BL $866 \times$ GM 8413(53.63 $\%)$. Whereas, only one cross SML $134 \times$ VGG 4 (51.75 \%) revealed positive significant heterosis over better parent

\section{Overall heterotic status of hybrids}

In addition to determining the overall general combining ability status of parents and overall specific combining ability status of crosses, it is also important to determine the overall heterotic status of the hybrids across the characters.

Hence, the same method which was used for determining overall sca status of hybrids was also utilized to compute overall heterotic status of each cross and the results of the same are tabulated in table 2. It is evident from the table that, 16 out of 28 hybrids manifested high $(\mathrm{H})$ overall heterotic status while the remaining 12 crosses expressed low (L) overall heterotic status across the traits.

As in the case of gca and sca, the magnitude and direction of heterosis varied considerably among the characters and therefore, it was difficult to decide the superiority of a hybrid over better parent. Hence, to decide whether a hybrid could be considered overall heterotic or not, the method proposed by Arunachalam and Bandyopadhyay (1979), with slight modification as by Mohan Rao (2001) was used. The results obtained clearly indicated high $(\mathrm{H})$ overall heterotic status of 16 hybrids, while the remaining 12 hybrids had low (L) heterotic status. The hybrids, $\mathrm{Hg}$ 19/A $\times$ NP 36, BL $866 \times$ VGG 4, LM $567 \times$ KKM 3 had highest total score over the final norm.

\section{Inter-relationship of overall gca status, sca status and heterotic status}

Based on the overall gca effects, the crosses were classified as $\mathrm{HH}$ (both the parents of the cross with high overall gca status), HL (female parent with high and the male parent with low overall gca status), LH (female parent with low and the male parent with high overall gca status) and LL (both the parents of a hybrid with low overall gca status). The relationship between overall gca and sca status (Table 3) clearly indicated that both the parents with low overall gca status $(\mathrm{L} \times \mathrm{L})$ also produced hybrids with high $(\mathrm{H})$ overall sca status and the hybrids with $\mathrm{H} \times \mathrm{H}$ gca combination of parents expressed high $(\mathrm{H})$ as well as low (L) overall sca status. Contrary to this, the hybrids involving high $(\mathrm{H}) \times$ low $(\mathrm{L})$ or low $(\mathrm{L}) \times$ high $(\mathrm{H})$ gca status expressed high $(\mathrm{H})$ overall sca status in higher frequency in most of the crosses indicating major role of non-additive gene action.

When both overall gca status and overall heterotic status was considered, it clearly indicated that the hybrids involving $\mathrm{H} \times \mathrm{H}$ parental combination produced high $(\mathrm{H})$ overall heterotic status, while, $\mathrm{L} \times \mathrm{L}$ parental combination produced low (L) overall heterotic status in the hybrids.

This suggested major role of additive gene action or additive $x$ additive interaction in such hybrids. However, the hybrids with $\mathrm{H} \times$ $\mathrm{L}$ or $\mathrm{L} \times \mathrm{H}$ type of parental combination always produced hybrids with high $(\mathrm{H})$ overall heterotic status, which indicated clearly the importance of non-additive gene action in the expression of heterosis. So these types of hybrid combinations are suitable for heterosis breeding. Whereas, the other 
combinations of $\mathrm{H} \times \mathrm{H}$ or $\mathrm{L} \times \mathrm{L}$ can be utilized to get useful transgressive segregants in the subsequent segregating generations.

In total, the overall heterotic status depends on the gca status of parents, the best combiners always produced best hybrids.

\section{References}

Arunachalam, V. and Bandopadhyaya, A., 1979, Are "Multiple cross- Multiple pollen hybrids" an answer for productive populations in Brassica compestris variety brown sarson. Theor. Appl. Genet., 54: 203-207.

Dethe A.M. and Patil J.V., 2008. Heterosis studies in mungbean (L.) Wilczek). Legume Res., (1): 36-39.

Gwande V.L., Patil J.V., Kute N.S., Dhole,V. and Patil D.K., 2001. Heterosis studies in mungbean (Vigna radiate (L.) Wilczek). New Botanist: 127-134.

Hayes, H. K., Immer, F. R. and Smith, D. C., 1955, Methods of Plant Breeding. Mc. Graw Hill Book Co., Inc., New York: 551Pp.

Joseph and Santosh Kumar, A.V., 2000) Heterosis and inbreeding depression in mungbean (Vigna radiata (L.) Wilczek). Legume Res., (2): 118-121.

Khattak, G. S. S., Haq, M. A., Ashraf, M., Marwat, E. U. K. and Srinivas, P., 2002e, Heterosis for seed yield and components in mungbean [Vigna radiata (L.) Wilczek]. Sci. Asia., 28:
345-350.

Kumar, U. M., Sheshshayee, M. S., Nataraj, K. N., Bindhu, M. H, Devendra, R. Aftab, H. I. S. and Prasad, T. G. 1998. Why has breeding for water use efficiency not been successful: An analysis and alternate approach to exploit this trait for crop improvement. Current Science 74: 994-1000.

Mohan Rao, A., 2001, Heterosis as a function of genetic divergence in sunflower (Helianthus annuus L.). Ph. D. thesis submitted to Acharya N. G. Ranga Agricultural University, Hyderabad, and 208pp.

Naidu N.V. and Satyanarayan A., 1993. Heterosis and combining ability in mungbean (Vigna radiata (L.)Wilczek). Indian J. Pulse Res., (1): 102-105.

Passioura, J. B. 1986, Resistance to drought and salinity: Avenues for improvement. Aust.J.Pl. Physiol., 13:191-201.

Panse, V. G. and Sukathme, P. V. 1967, Statistical Methods for Agricultural Workers, ICAR, New Delhi, pp 145.

Thomas, Robertson, M. J., Fulakai and Peoples, M. B, 2004, the effect of timing and severity of water deficit on growth, development, yield accumulation and nitrogen fixation of mungbean. Field Crops Res., 86: 67-80.

Turner, J. H., 1953, a study of heterosis in upland cotton-I, Yield of hybrids compared with varieties, Agron. J., 45: 487-490.

\section{How to cite this article:}

Sheela Duddagi and Savithramma, D.L. 2017. Heterosis Study for Yield, Water Use Efficiency and its Attributing Traits in Greengram (Vigna radiata L. Wilczek). Int.J.Curr.Microbiol.App.Sci. 6(7): 2705-2715. doi: https://doi.org/10.20546/ijcmas.2017.607.380 